\title{
Staying Connected to your Athletes During COVID-19 Through Virtual Training
}

Glenn Corcoran, MExSc (S\&C), BExSc (Rehab), CSCS, RSCC*E1 ${ }^{1}$ Rick Martin, Dip Fitness, ASCA L3 PCAS-M ${ }^{1}$, Patricia Sullivan, ASCA L12

${ }^{1}$ High Performance Training Centre, Bond University, Gold Coast, QLD, Australia

${ }^{2}$ Bond University Rugby Club, Gold Coast, QLD, Australia

\section{CHALLENGING TIMES}

March 2020 saw the dramatic abrupt halt of the Australian sport season due to COVID-19 and Australian Government sanctioned Social Distancing restrictions that followed. Our Premier rugby team had been training diligently at the Bond University High Performance Training Centre (HPTC) and was one week away from the start of their 2020 opening game. HPTC squads such as netball, athletics and swimming were already preparing for competition mode, state and national championships, the results of which would determine team selection and qualifying for Athletics championships and Olympic Games as their major focal points. The teams and athletes we had been preparing were excited for 2020 as our testing results and state championship results had been better than previous preparation periods and expectations for the season were high for all.

\section{THE PROBLEM}

The issues that impacted directly and immediately were the social distancing, lock down of training facilities and the impending problem of losing connections with athletes that were driven to succeed, primed to compete in peak physical condition and limited options to continue training as they know it. It was our priority to keep athletes and coaches connected and our teams were quick to implement strategies to address these issues from the outset. The HPTC in collaboration with Bond University Sport also reacted expediently to address the needs of our athletes and implement plans from the very first week of lockdown.

We identified the problem was keeping highly motivated athletes engaged. All athletes were either close to beginning a season they had trained for with highly structured post-season, off-season, preseason and pre-competition phases, achieving the targets set for them individually and as teams. Everything now had changed quickly with minimal warning. The key question here is how do we maintain these connections with the athletes, coaches, and clubs when the physical and mental issues they were about to face had become reality?

This brief summary outlines how we are staying connected to your athletes during covid-19 through virtual training though 
the What, Where, When, Why and How approach. We will focus predominantly on the physical strategies that we could directly impact through the HPTC.

\section{THE BOND HPTC SOLUTION}

What - did we see as issue?

The issue for us was highly trained athletes in peak condition immediately communicated that the competitions were cancelled or postponed indefinitely. Their structured schedules around training in the gym, conditioning sessions, recovery protocols and interactions with coaches, strength and conditioning ( $S \& C$ ), social connections around their clubs, work and social lives potentially would be significantly diminished.

Where - did we set up to address the issue

We believed it was critical to act swiftly before the athletes' potential mental states deteriorated. It was crucial to provide clarity and confidence around the areas we could impact on as professional and experienced S\&C coaches with the HPTC as our venue. Adhering to social distancing rules we decided to implement training options for all our athletes by developing an extensive ongoing video training format ensuring the athletes were aware they had our full support. The goal was to provide the best possible physical preparation with the resources at our disposal. In the event lockdown restrictions were lifted our athletes would be prepared to resume full weight room lifting and team field training sessions allowing for a smoother transition into the new competitive season with the decreased risk of potential injury concerns. We set up the HPTC, as it is closed to all training, to have an area that we could video all ranges of exercise and training options so our teams and athletes could have a resource library of routines to work on during isolation.

\section{When - what time frames did we implement}

We implemented and sent out via email and social networking platforms, training sessions which include timed runs, weights and stretching sessions within the first week of lockdown.

Our focus during that first week was to design well-formatted professional videos, including world's best practice, content, and structure, with research into the platforms that maximise engagement for our athletes, to then roll out the finished product. At the start of week two we had numerous completed videos ready for distribution to our athletes and teams. By the end of week two we had started delivering completed products to our athletes on a regular but strategic basis. Full time HPTC staff are available to discuss or explain any on the content provided via our standard communication channels.

\section{Why - was this strategy from HPTC important}

The clubs and coaches had implemented numerous strategies to keep the athletes engaged with leadership groups, challenges, and coaching opportunities that were implemented quickly and professionally. We knew there was a fantastic opportunity to assist with these connections for athletes by delivering in the strength and condition landscape. This would assist our colleagues in helping to keep positivity around themselves, coaches, and club engagement. Structure 
and routine around physical preparation is an important part of their lives as athletes and who they are, motivated and driven individuals. We knew this was an area we could address that could have profound positive outcomes on physical readiness and thus mental wellbeing as part of Bond University Sports overall strategy. The strategy was also a critical factor to ensure athletes still felt valued, as many of our Sports organisations (national and state) have not implemented training options of significant content to grass roots teams and clubs. This was an ideal opportunity to engage these groups and grow relationships that can be under stress already. I feel these large organisations that had a great opportunity to give educational content back to their grass roots base at minimal cost to produce and implement have missed the boat. Many have decided to post content of poor quality with little significance and that is an opportunity lost.

Once we implemented the initial two weeks of session videos to our HPTC connected athletes and squads, Bond Sport approached us to see if we would make the content available to feeder associations to our premier squads in rugby and netball, and the uptake and feedback has been impressive.

How - we addressed and implemented the program

Compromising quality and content was not an option, so dedicated an area in the gym that met the social distancing requirements with camera person and the S\&C in the centre and shot the video's with Sony a6400 mirrorless camera (1) that allowed high quality content. We ensured from the embryotic stage that all content would provide both professional and technical consistency to our client base.

The selected area captured routines completed by our coaches, in professional attire so there was familiarity of surrounds and the content was designed and delivered by HPTC and Bond Rugby staff. A primary goal was to confirm to our athletes that we are committed to them. The HPTC livestreams a number or Yoga, Pilates and Tai Chi sessions three times a week at 6:00am to ensure athletes keep a routine and begin their day in a positive mindset. Several of these sessions have in excess of 100 athletes participating and we target set clubs for these sessions to consolidate that team culture. The commitment to our athletes through the lockdown will be ongoing and we have currently completed videos as below:

- Bodyweight training sessions

- Warmup routines

- Yoga / Pilates / Tai Chi

- Stretching sessions and Band work

- Abdominal sessions

- BW Circuits

- Footwork and Speed drills

- Change of Direction

- Breathing techniques

- Myofascial release work

- Numerous other sessions in the mix and ready to implement

The video content for bodyweight training sessions aligns with the NSCA guidelines in relation to bodyweight training methods (2). The videos are then uploaded to the Facebook pages of all clubs. Feeder Clubs and aligned associations have set up their own private staff and athlete resource pages where we upload the content. In the initial seven weeks of the lockdown we have produced $>25$ recorded sessions with content going to over 1200 athletes within 
Gold Coast, Australia and selected videos made available to Bond Sport and Bond Staff with a reach of another 5,000 people.

\section{References}

1. Sony. https://www.techradar.com/au/reviews/sonyalpha-a6400-review

2. Haff, G.G., Berninger, D., \& Caulfield, S (2016). Exercise Technique for Alternative Modes and Nontraditional Implement Training. In G.G. Haff \& N.T. Triplett (Eds), Essentials of Strength Training and Conditioning (pp.409438). Champaign, Illinois: Human Kinetics. 